\title{
Valor pronóstico del sistema de calificación APACHE II en el postoperatorio de cirugía cardíaca
}

\author{
Prognostic value of APACHE II score in postoperative of cardiac surgery \\ Leonardo A. Seoane ${ }^{*}$, Juan Espinoza², Lucrecia Burgos ${ }^{3}$ Juan Furmento ${ }^{1}$, Luis Polero ${ }^{3}$, \\ Mariano Camporrotondo², Mariano Vrancic ${ }^{2}$, Daniel Navia² y Mariano Benzadón ${ }^{1}$ \\ ${ }^{1}$ Servicio de Cardiología, Sección de Cardiología Crítica y Recuperación Cardiovascular; ${ }^{2}$ Servicio de Cirugía Cardiovascular; ${ }^{3}$ Servicio de \\ Cardiología. Instituto Cardiovascular de Buenos Aires (ICBA), Buenos Aires, Argentina
}

\begin{abstract}
Resumen
Antecedentes y objetivos: El sistema de calificación APACHE II permite predecir la mortalidad intrahospitalaria en terapia intensiva. Sin embargo, no está validado para cirugía cardíaca, ya que no posee buena capacidad diferenciadora. El objetivo es determinar el valor pronóstico de APACHE II en el postoperatorio de procedimientos cardíacos. Materiales y métodos: Se analizó en forma retrospectiva la base de cirugía cardíaca. Se incluyó a pacientes intervenidos entre 2017 y 2018, de los cuales se calculó la puntuación APACHE II. Se utilizó curva ROC para determinar el mejor valor de corte. El punto final primario fue mortalidad intrahospitalaria. Como puntos finales secundarios se evaluó la incidencia de bajo gasto cardíaco (BGC), accidente cerebrovascular ( $A C V$ ), sangrado quirúrgico y necesidad de diálisis. Se realizó un modelo de regresión logístico multivariado para ajustar a las variables de interés. Resultados: Se analizó a 559 pacientes. La media del sistema de calificación APACHE Il fue de 9.9 (DE 4). La prevalencia de mortalidad intrahospitalaria global fue de 6.1\%. El mejor valor de corte de la calificación para predecir mortalidad fue de 12, con un área bajo la curva ROC de 0.92. Los pacientes con APACHE II $\geq 12$ tuvieron significativamente mayor mortalidad, incidencia de BGC, ACV, sangrado quirúrgico y necesidad de diálisis. En un modelo multivariado, el sistema APACHE II se relacionó de modo independiente con mayor tasa de mortalidad intrahospitalaria (OR, 1.14; IC95\%, 1.08-1.21; $p$ < 0.0001). Conclusiones: El sistema de clasificación APACHE II demostró ser un predictor independiente de mortalidad intrahospitalaria en pacientes que cursan el postoperatorio de cirugía cardíaca.
\end{abstract}

Palabras clave: APACHE II. Cirugía cardíaca. Calificaciones del riesgo.

\begin{abstract}
Background and objectives: The APACHE I/ score allows predicting in-hospital mortality in patients admitted to intensive care units. However, it is not validated for patients undergoing cardiac surgery, since it does not have a good discriminatory capacity in this clinical scenario. The aim of this study is to determine prognostic value of APACHE II score in postoperative of cardiac surgery. Materials and methods: The study was performed using the cardiac surgery database. Patients undergoing surgery between 2017 and 2018, with APACHE II score calculated at the admission, were included. The ROC curve
\end{abstract}

\section{Correspondencia:}

*Leonardo A. Seoane

E-mail: laseoane@icba.com.ar
Fecha de recepción: 25-09-2019

Fecha de aceptación: 16-04-2020

DOI: 10.24875/ACM.20000349
Disponible en internet: $23-12-2020$ Arch Cardiol Mex. 2020;90(4):398-405 www.archivoscardiologia.com 1405-9940 / C 2020 Instituto Nacional de Cardiología Ignacio Chávez. Publicado por Permanyer. Este es un artículo open access bajo la licencia CC BY-NC-ND (http://creativecommons.org/licenses/by-nc-nd/4.0/). 
was used to determine a cut-off value The primary endpoint was in-hospital death. Secondary endpoints included low cardiac output (LCO), stroke, surgical bleeding, and dialysis requirement. A multivariable logistic regression model was developed to adjust to various variables of interest. Results: The study evaluated 559 patients undergoing cardiac surgery. The mean of APACHE II Score was 9.9 (SD 4). The prevalence of in-hospital death was 6.1\%. The best prognostic cut-off value for the primary endpoint was 12, with a ROC curve of 0.92. Patients with an APACHE II score greater than or equal to 12 had significantly higher mortality, higher incidence of $L C O$, stroke, surgical bleeding and dialysis requirement. In a multivariate logistic regression model, the APACHE II score was independently associated with higher in-hospital death (OR, 1.14; 95Cl\%, 1.08-1.21; $p<0.0001)$. Conclusions: The APACHE II Score proved to be an independent predictor of in-hospital death in patients undergoing postoperative cardiac surgery, with a high capacity for discrimination.

Key words: APACHE II Score. Cardiac surgery. Risk scores.

\section{Introducción}

En la actualidad, en las unidades de terapia intensiva, los pacientes críticos muestran una morbimortalidad intrahospitalaria elevada ${ }^{1,2}$. Esto puede deberse a la gravedad de la enfermedad, la complejidad de la operación o la condición previa del huésped, y puede ocasionar durante el internamiento disfunción de uno o más órganos ${ }^{1-3}$. La estratificación del riesgo de pacientes críticos es de gran importancia y ha sido objeto de estudio durante varias décadas. Desde 1970 se han utilizado distintos modelos de riesgo en las unidades de terapia intensiva para predecir episodios intrahospitalarios. Sin embargo, no es claro cuál es el mejor sistema de calificación para determinar el valor pronóstico de los pacientes internados en las distintas unidades críticas (sean generales, quirúrgicas o específicas, como la recuperación cardiovascular en la que se asiste de modo exclusivo a los enfermos postoperatorios de intervenciones cardíacas).

Existen diversos modelos que hacen posible predecir la mortalidad intrahospitalaria, a partir de variables calculadas al ingreso a la unidad de terapia intensiva. El APACHE (Acute Physiology and Chronic Health Evaluation) II, APACHE III, APACHE IV, SAPS (Simplified Acute Physiology Score) II, SAPS III y MPM (Mortality Probability Models) II son algunos de los sistemas de pronóstico utilizados habitualmente en terapia intensiva; por su parte, el SOFA (Sequential Organ Failure Assessment) y el MODS (Multiple Organ Disfunction) permiten evaluar la disfunción multiorgánica-11. La mayor parte de ellos se ha validado en poblaciones de unidades de terapia intensiva general, y no en unidades de terapias especiales o en ciertos subgrupos de pacientes.

Se ha excluido a los sujetos sometidos a operaciones cardíacas prácticamente de la mayoría de los trabajos que evaluaron los modelos de gradación del riesgo en pacientes críticos. Las unidades de recuperación cardiovascular y terapias intensivas de cirugía cardíaca atienden a una población específica, con características propias y singulares. Las consecuencias fisiopatológicas de la bomba de circulación extracorpórea modifican, aunque sea de manera transitoria, múltiples variables empleadas por lo regular por los sistemas de calificación de estratificación del riesgo en el postoperatorio inmediato. Además, se han observado múltiples cambios fisiológicos debido a la utilización de dispositivos como el balón de contrapulsación intraaórtico, la hemofiltración, la ventilación mecánica y la asistencia ventricular, que dificultan la estratificación con los modelos de riesgo comunes ${ }^{12}$.

Por tal motivo, en el ámbito de la cirugía cardíaca, los principales parámetros usados hoy en día para la gradación del riesgo quirúrgico son el EuroSCORE II y el STS ${ }^{13,14}$. Si bien son modelos valiosos y validados para predecir morbimortalidad en pacientes sometidos a operación cardíaca, ambos usan variables evaluadas de manera preoperatoria. Por lo tanto, no consideran diversos factores intraoperatorios o postoperatorios inmediatos de relevancia, que son determinantes para el pronóstico de los pacientes.

Respecto de los biomarcadores postoperatorios de cirugía cardíaca, existen predictores habituales con valor pronóstico, como el ácido láctico y la saturación venosa de oxígeno. Pese a que son buenos indicadores de hipoperfusión tisular que permiten guiar una terapéutica, no pueden predecir de manera aislada el riesgo real del paciente ${ }^{15-20}$.

En relación con los sistemas de calificación del riesgo empleados en pacientes críticos, el APACHE II es aún la norma de referencia para predecir mortalidad intrahospitalaria en unidades de terapia intensiva, ya que posee amplia evidencia en unidades críticas médicas y quirúrgicas, es simple de calcular, se basa en variables fisiológicas, tiene más de 30 años de uso y se ha validado en múltiples cohortes de diferentes países, con buena capacidad de diferenciación en los 
distintos escenarios clínicos en los que se ha evaluado 4,21-27. Sin embargo, debido a la sobreestimación del riesgo calculado, no se ha validado en pacientes posquirúrgicos cardíacos.

El objetivo principal del estudio fue determinar el valor pronóstico del sistema de calificación APACHE ॥ en individuos que cursan el postoperatorio de cirugía cardíaca en un centro cardiovascular de alta complejidad.

\section{Métodos}

Se realizó un estudio retrospectivo, observacional y transversal de problemas de comparación de factores de riesgo. Se analizó la base de datos del servicio de cirugía cardíaca, completada de manera prospectiva. Se incluyó a los pacientes sometidos a intervención quirúrgica cardíaca en el Instituto Cardiovascular de Buenos Aires, Argentina, entre abril de 2018 y marzo de 2019, y de quienes se determinó la puntuación de APACHE II al ingresar a la unidad de terapia intensiva.

Fueron elegibles para su inclusión todos los pacientes de edad $\geq 18$ años, objeto de alguna de las siguientes operaciones cardiovasculares, ya sea de manera electiva o de urgencia: cirugía de revascularización miocárdica (CRM), operación valvular (aórtica, mitral, tricuspídea, o pulmonar), operación de cayado aórtico o aorta ascendente (reemplazo aorto-aórtico, técnica de Bentall de Bono, Tyrone David o Yacoub), operación de reconstrucción ventricular, miectomía septal, corrección de cardiopatía congénita, operación de Maze, trasplante cardíaco, o intervenciones cardíacas combinadas (dos o más de los procedimientos ya señalados), y con calificación APACHE II calculada al ingresar a la terapia intensiva. Se excluyó a los pacientes que no tenían puntuación de APACHE II a las 24 horas tras el ingreso a terapia intensiva, o que manifestaran su negativa de participar.

La variable independiente del estudio fue la calificación de gravedad APACHE II, que se calculó al ingreso del paciente a la unidad de terapia intensiva y a partir de distintas variables: edad, temperatura rectal, presión arterial media, frecuencia cardíaca, frecuencia respiratoria, oxigenación (se utilizó la presión arterial de oxígeno si la fracción inspirada de oxígeno era $<50 \%$ o el gradiente alveolar arterial de oxígeno $>50 \%$ ), $\mathrm{pH}$ arterial, valores de sodio y potasio, creatinina plasmática, hematócrito, recuento de leucocitos, escala de Glasgow, y presencia de enfermedad crónica. El intervalo de la calificación pudo variar entre 0 y 71 . Se evaluó de modo inicial como variable proporcional continua. Luego de obtener el mejor valor de corte para predecir el episodio primario, se utilizó también como variable dicotómica para el análisis multivariado.

Como variables dependientes a analizar se incluyeron las siguientes:

1. Mortalidad intrahospitalaria: La mortalidad que ocurrió durante el mismo internamiento en el que se practicó la operación cardíaca.

2.Bajo gasto cardíaco (BGC): Se definió como la presión arterial sistólica $<90 \mathrm{mmHg}$ con requerimiento de inotrópicos, junto con un índice cardíaco $<2.2 \mathrm{~L} / \mathrm{min} / \mathrm{m}^{2}$ medido por termodilución con catéter de Swan-Ganz, y con presión de enclavamiento pulmonar $>18 \mathrm{mmHg}$.

3. Accidente cerebrovascular (ACV): Se definió como foco neurológico nuevo, transitorio o permanente, en relación con una nueva imagen isquémica o hemorrágica en la tomografía computarizada cerebral.

4.Sangrado quirúrgico: Hemorragia postoperatoria con requerimiento de medidas quirúrgicas para su control, ya sea por sangrado excesivo, hemotórax o taponamiento cardíaco.

5. Insuficiencia renal con necesidad de diálisis: Requerimiento de diálisis por incremento de creatinina plasmática tres veces sobre el valor basal junto con oliguria o anuria, o por presencia de criterios de urgencia: hiperpotasemia o acidosis resistente, sobrecarga de volumen, encefalitis urémica o pericarditis urémica.

El estudio cumplió todos los requerimientos contenidos en el código ético de la OMS (Declaración de Helsinki) y recibió aprobación del Comité de Investigación Clínica del instituto y el Comité de Ética. Todos los pacientes firmaron el Habeas data.

\section{Análisis estadístico}

La distribución paramétrica de las variables continuas cuantitativas se evaluó mediante la prueba de Kolmogorov-Smirnov. Las variables cualitativas se expresaron en proporciones, mientras que las cuantitativas continuas en medias, con sus respectivas desviaciones estándar (DE) (en caso de distribución paramétrica), o medianas y rango intercuartílico para las no paramétricas.

Se utilizó la prueba de T de Student para el análisis de variables cuantitativas de distribución paramétrica y la $U$ de Mann-Whitney en caso de las no paramétricas. La relación entre variables cualitativas se definió a partir de las pruebas de $\chi^{2}$ y Fisher.

El punto final primario analizado fue la mortalidad intrahospitalaria. Como puntos finales secundarios se 
evaluó la prevalencia de BGC, ACV, sangrado quirúrgico y necesidad de diálisis.

Para determinar la calibración de APACHE II se recurrió a la prueba de bondad de ajuste de Hosmer-Lemeshow, que dividió a la población en deciles de acuerdo con las probabilidades de muerte previstas $y$ se obtuvo una $\chi^{2}$ a partir de las frecuencias observadas y esperadas. Una $p>0.05$ se consideró como un buen ajuste. Se utilizó luego la curva ROC para definir la capacidad de diferenciación de la calificación APACHE II e identificar el mejor valor de corte para predecir mortalidad intrahospitalaria.

Se realizó un modelo de regresión logístico multivariado para ajustar a diversas variables de interés. Se presentaron razones de momios (OR, odds ratio) con sus respectivos intervalos de confianza de $95 \%$ para las variables incluidas en los modelos. Se consideró un error $\alpha$ de dos colas del 5\% como valor estadísticamente significativo $(p<0.05)$.

Los análisis estadísticos se realizaron con el software IBM SPSS Statistics versión 19.0.

\section{Resultados}

Se analizó a 559 pacientes sometidos a operación cardíaca. Del total, $48 \%$ correspondió a cirugía de revascularización miocárdica (CRM), $24 \%$ a operación valvular, $15 \%$ a combinada (operación valvular relacionada con CRM) y $13 \%$ con otro tipo de intervención. La media de edad fue de 67.1 años (DE 10) y la mayoría fue del sexo masculino (73.4\%). Respecto de los factores de riesgo cardiovascular, $76 \%$ correspondió a hipertensos, $24 \%$ a diabéticos, $50 \%$ a fumadores y $6 \%$ tenía antecedentes heredofamiliares. En relación con la presencia de otras comorbilidades, $9 \%$ mostraba antecedentes de fibrilación auricular, 14\% de disfunción ventricular moderada/grave, $7 \%$ de operación cardíaca, $6 \%$ de enfermedad pulmonar crónica, $3 \%$ de accidente cerebrovascular previo y $8 \%$ de insuficiencia renal crónica. En cuanto al procedimiento cardíaco practicado, en el $53 \%$ de los casos se utilizó bomba de circulación extracorpórea y el $25 \%$ fue de urgencia. La media global de la calificación APACHE II fue de 7.9 (DE 4).

Al efectuar un análisis de las características basales, y dividir a la población según el mejor valor de corte de APACHE II para predecir mortalidad intrahospitalaria, se observó que aquellos que presentaron mayor valor de APACHE II se caracterizaron por ser significativamente más añosos, tener menor índice de masa corporal, mayor tasa de insuficiencia renal crónica, fibrilación auricular, operación cardíaca previa y
Tabla 1. Características basales de la población dividida de acuerdo con el mejor valor de corte del sistema APACHE II para predecir mortalidad intrahospitalaria (APACHE: 12)

\begin{tabular}{|c|c|c|c|c|c|}
\hline APACHE II & \multicolumn{2}{|c|}{$<12$} & \multicolumn{2}{|c|}{$\geq 12$} & \multirow[t]{2}{*}{$\mathbf{P}$} \\
\hline Variables & Media & DE & Media & DE & \\
\hline Edad (años) & 65.9 & 11.0 & 70.0 & 10.6 & 0.0001 \\
\hline Sexo femenino & $25.5 \%$ & & $32.8 \%$ & & 0.069 \\
\hline Peso $(\mathrm{kg})$ & 82.4 & 14.8 & 75.9 & 12.9 & 0.0001 \\
\hline Altura $(\mathrm{cm})$ & 169.9 & 9.3 & 166.6 & 9.0 & 0.0001 \\
\hline HTA & $77.7 \%$ & & $73.1 \%$ & & 0.226 \\
\hline TB0 & $53.4 \%$ & & $44.1 \%$ & & 0.039 \\
\hline Diabetes & $24.1 \%$ & & $23.1 \%$ & & 0.792 \\
\hline AHF & $6.7 \%$ & & $5.4 \%$ & & 0.542 \\
\hline $\begin{array}{l}\text { Insuficiencia } \\
\text { renal crónica }\end{array}$ & $5.6 \%$ & & $15.1 \%$ & & 0.0001 \\
\hline $\begin{array}{l}\text { Enfermedad } \\
\text { pulmonar crónica }\end{array}$ & $5.1 \%$ & & $8.6 \%$ & & 0.107 \\
\hline ACV & $2.7 \%$ & & $5.4 \%$ & & 0.106 \\
\hline Ritmo sinusal & $96.5 \%$ & & $82.3 \%$ & & 0.0001 \\
\hline $\begin{array}{l}\text { Operación de } \\
\text { urgencia }\end{array}$ & $22.0 \%$ & & $37.1 \%$ & & 0.0001 \\
\hline $\begin{array}{l}\text { Utilización de } \\
\text { CEC }\end{array}$ & $49.8 \%$ & & $68.5 \%$ & & 0.0001 \\
\hline $\begin{array}{l}\text { Operación } \\
\text { cardíaca previa }\end{array}$ & $4.3 \%$ & & $12.4 \%$ & & 0.0001 \\
\hline FSVI Mod/Gra & $10.5 \%$ & & $20.4 \%$ & & 0.001 \\
\hline APACHE II & 7.5 & 2.2 & 16.4 & 9.0 & 0.0001 \\
\hline
\end{tabular}

ACV: accidente cerebrovascular; AHF: antecedentes heredofamiliares; CEC: bomba de circulación extracorpórea; DE: desviación estándar; FSVI Mod/Gra: disfunción ventricular izquierda moderada/grave; HTA: hipertensión arterial; TBQ: tabaquismo.

disfunción ventricular izquierda (tabla 1). La población con puntuación de APACHE $\| \geq 12$ registró además mayor porcentaje de utilización de la bomba de circulación extracorpórea y mayor tasa de operaciones de urgencia.

La prevalencia de mortalidad intrahospitalaria global fue de $6.1 \%$. En relación con los puntos finales secundarios, se observó una tasa de sangrado quirúrgico de 9.3\%, BGC del $12.7 \%$, ACV del $1.6 \%$ y de insuficiencia renal con requerimiento de diálisis de $4.1 \%$.

La prueba de bondad de ajuste de Hosmer-Lemeshow reveló un valor de $p>0.05$, lo que implica una buena calibración de la calificación para dicha población 


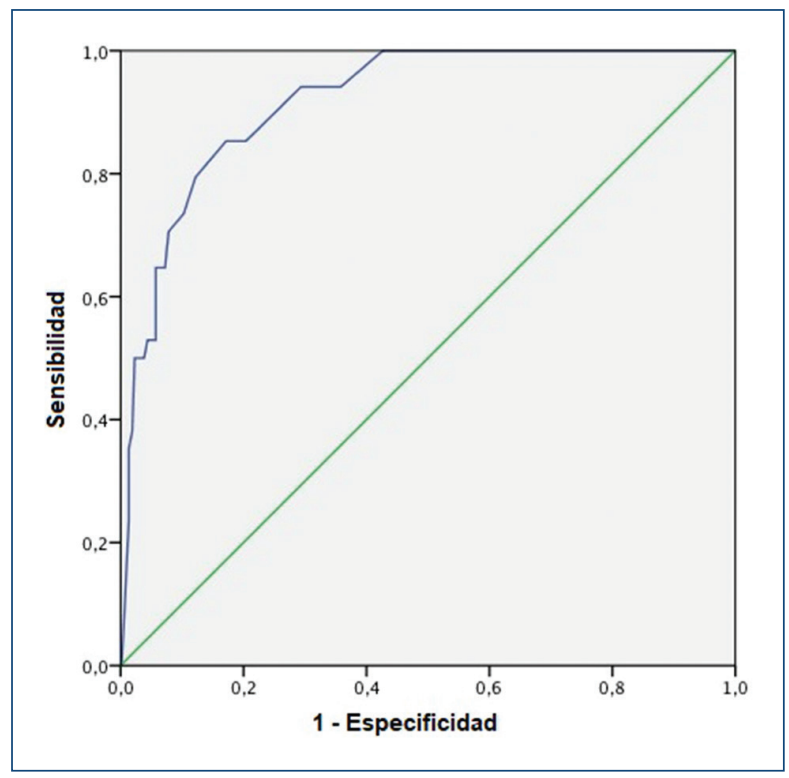

Figura 1. Curva ROC del sistema de calificación APACHE II para predecir mortalidad intrahospitalaria. La puntuación APACHE II tuvo un área bajo la curva ROC de 0.92 (IC95\%, $0.88-0.96 ; p=0.0001$ ), con una sensibilidad del $85 \%$ y una especificidad del $80 \%$.

$\left(\chi^{2}=2.1 ;\right.$ grado de libertad $\left.=8 ; p=0.90\right)$. La puntuación APACHE II mostró además una muy buena capacidad de diferenciación para predecir mortalidad intrahospitalaria, con un área bajo la curva ROC de 0.92 (IC95\%, $0.88-0.96 ; p=0.0001$ ), con una sensibilidad del $85 \%$ y una especificidad del $80 \%$ (Fig. 1). El mejor valor de corte de la puntuación obtenida por curva ROC para predecir el episodio final primario fue de 12. Los pacientes con un valor de APACHE II $\geq 12$ presentaron significativamente mayor mortalidad intrahospitalaria, mayor incidencia de BGC, ACV, sangrado quirúrgico y necesidad de diálisis (tabla 2).

En un modelo de regresión logística multivariado, en el cual se ajustó a diversas variables (sexo, peso, tabaquismo, antecedente de accidente cerebrovascular, insuficiencia renal crónica, fibrilación auricular, operación cardíaca previa, disfunción ventricular, y utilización de bomba extracorpórea), cada punto de aumento en la calificación APACHE II se vinculó significativamente con mayor tasa de mortalidad intrahospitalaria (OR, 1.14; IC95\%, 1.08-1.21; $p<0.0001$; tabla 3). Si se toma la variable del APACHE II como dicotómica a partir del mejor valor de corte, se observó que presentar un valor de la puntuación > 12 también se vinculó de manera independiente y significativa con mayor tasa de mortalidad (OR, 36.5; IC95\%, 8.2-161.6; $p<0.0001$ ).
Tabla 2. Puntos finales (primarios y secundarios) evaluados de acuerdo con el valor del APACHE II

\begin{tabular}{|l|c|c|c|c|}
\hline Episodio & $\begin{array}{c}\text { APACHE } \\
<\mathbf{1 2}\end{array}$ & $\begin{array}{c}\text { APACHE } \\
\geq \mathbf{1 2}\end{array}$ & Total & $\mathbf{p}$ \\
\hline $\mathrm{N}$ & 373 & 186 & 559 & \\
\hline Mortalidad & $0.5 \%$ & $17.2 \%$ & $6.1 \%$ & $<0.0001$ \\
\hline Sangrado quirúrgico & $1.9 \%$ & $24.2 \%$ & $9.3 \%$ & $<0.0001$ \\
\hline BGC & $0.3 \%$ & $37.6 \%$ & $12.7 \%$ & $<0.0001$ \\
\hline Diálisis & $0 \%$ & $12.4 \%$ & $4.1 \%$ & $<0.0001$ \\
\hline ACV & $0.8 \%$ & $3.2 \%$ & $1.6 \%$ & 0.032 \\
\hline
\end{tabular}

ACV: accidente cerebrovascular; BGC: bajo gasto cardíaco; n: número de pacientes.

Tabla 3. Modelo de regresión logística multivariado para predecir mortalidad intrahospitalaria

\begin{tabular}{|l|c|c|c|c|}
\hline & \multirow{2}{*}{ Variables } & OR & \multicolumn{2}{|c|}{ IC95\% } \\
\cline { 4 - 5 } & & & Inferior & Superior \\
\hline $\begin{array}{l}\text { Insuficiencia renal } \\
\text { crónica }\end{array}$ & 0.001 & 4.001 & 1.394 & 9.693 \\
\hline CEC & & & & \\
\hline Sexo femenino & 0.024 & 5.016 & 1.242 & 20.251 \\
\hline Fibrilación auricular & 0.003 & 4.223 & 1.632 & 10.930 \\
\hline ACV previo & 0.008 & 9.517 & 1.812 & 49.996 \\
\hline TB0 & 0.033 & 2.750 & 1.087 & 6.957 \\
\hline $\begin{array}{l}\text { Operación cardíaca } \\
\text { previa }\end{array}$ & 0.002 & 3.256 & 1.549 & 7.382 \\
\hline FSVI Mod/Gra & 0.0001 & 1.542 & 1.213 & 1.840 \\
\hline $\begin{array}{l}\text { APACHE II } \\
\text { (por c/punto) }\end{array}$ & 0.0001 & 1.141 & 1.076 & 1.211 \\
\hline
\end{tabular}

ACV: accidente cerebrovascular; CEC: bomba de circulación extracorpórea; FSVI Mod/Gra: disfunción ventricular izquierda moderada/grave; OR: razón de momios; TBQ: tabaquismo.

\section{Discusión}

En la actualidad, predecir mortalidad en las unidades de terapia intensiva es fundamental y un estándar de calidad $^{28,29}$. Los pacientes que cursan el postoperatorio de una intervención cardíaca son una población especial, ya que los distintos modelos predictores habitualmente utilizados en áreas críticas no son exactos, en virtud de sus características perioperatorias, y sobreestiman la mortalidad. Sin embargo, en este estudio, el sistema de calificación APACHE II demostró tener 
una adecuada calibración y diferenciación, con muy buena capacidad para predecir mortalidad intrahospitalaria en pacientes postoperatorios de procedimientos cardíacos en la unidad de terapia intensiva de un centro cardiovascular de alta complejidad.

En comparación con los trabajos que evaluaron la puntuación APACHE II en las unidades de terapia intensiva general de distintos países, la población evaluada fue distinta. En los estudios conducidos en unidades terapias intensivas generales se incluyó a pacientes más jóvenes, con una media de 44 años en los protocolos de Jacobs, et al., de 53 en el de Teik, et al., y de 58 años en el estudio canadiense de Wong, et al ${ }^{22,23,26,27}$. En contraste, la media de la población que se analizó en esta investigación fue mayor (67.1 años). Las comorbilidades de la población fueron sustancialmente distintas dado que, en las unidades críticas generales, los pacientes tuvieron en particular mayores antecedentes renales y respiratorios (37 vs. 6\%), pero menor porcentaje de disfunción ventricular izquierda ${ }^{23}$. En promedio, la mitad de los pacientes ingresó a la unidad de terapia intensiva por alteración quirúrgica (la mayoría operaciones no cardíacas), en tanto que en este trabajo todos cursaban el postoperatorio de un procedimiento quirúrgico cardíaco. La electividad de la operación fue heterogénea, en algunos casos similar a la de este estudio ( $25 \%$ de emergencia), pero en algunos casos el porcentaje fue mayor $(32 \%)^{22,26}$.

En relación con los estudios que evaluaron calificaciones específicas para sujetos sometidos a intervención cardíaca, como el CASUS (Cardiac Surgery Score) y el POCAS (Post Cardiac Surgery Score); las características basales poblacionales fueron similares a las de este trabajo ${ }^{30-33}$. La principal diferencia observada en el protocolo original de Hekmat, et al. fue la inclusión de mayor porcentaje de CRM aisladas (62 vs. $48 \%$ ) y el menor número de procedimientos valvulares $(16.5 \text { vs. } 24 \%)^{30}$.

En este trabajo, la media global del APACHE II fue de 7.9 (DE 4). En comparación con otras cohortes que analizaron unidades de terapia intensiva generales, el valor fue bajo. La media varió desde cifras de 13 en las cohortes de Arabia Saudita, 14.2 en EE.UU., 14.7 en Japón, 16.5 en Canadá y 17.9 en Reino Unido hasta 20.1 en China ${ }^{4,22-27}$. Es probable que esta diferencia se debiera a que los pacientes que cursaron el posoperatorio de intervención cardíaca tuvieron menos comorbilidades y mejor puntuación fisiológica al ingresar a la unidad crítica y por tanto el riesgo estaba determinado sobre todo por la intervención quirúrgica misma. No obstante, cuando se compara la media del APACHE II con estudios que evaluaron sólo a sujetos con operaciones cardíacas (como el de Hekmat, et al.), la media fue similar (8 vs. 7.9$)^{30}$.

La mortalidad intrahospitalaria global observada fue de $6.1 \%$. En contraposición, en los análisis efectuados en unidades de terapia intensiva generales, la tasa fue significativamente mayor. Ésta varió desde $16.9 \%$ en Japón, 19.7 en Estados Unidos, 24.8\% en Canadá, $27 \%$ en Reino Unido y $36 \%$ en China hasta $46 \%$ en Arabia Saudita ${ }^{4,22-27}$. Desde luego, la diferencia se explica porque en este estudio se incluyó sólo a individuos sometidos a operación cardíaca en un centro cardiovascular de alta complejidad, con menores comorbilidades respecto de aquéllos asistidos en las unidades de terapia intensiva generales. Si bien la tasa fue baja, el valor del sistema de calificación APACHE Il también fue bajo, con una adecuada concordancia entre la mortalidad esperada y la real.

Al analizar estudios que consideraron a pacientes postoperatorios de procedimientos quirúrgicos cardíacos, la mortalidad fue similar a la registrada en esta investigación: Badreldin, et al. (5.8\%) y Exarchopoulos, et al. $(6.0 \%)^{17,32}$. Pese a ello, en otros grupos se reconocieron diferencias, como en el de Tamayo, et al., que mostró una mortalidad mayor $(9.0 \%)^{33}$. En cambio, Hekmat, et al. publicaron en su cohorte una mortalidad menor a la de este protocolo $(4.4 \%)^{30}$. Esta diferencia puede deberse a la menor complejidad de las operaciones cardíacas que incluyó a la cohorte alemana, ya que tuvo mayor número de CRM y menor tasa de intervenciones valvulares.

En cuanto a la calibración del sistema APACHE II a través de la prueba de Hosmer-Lemeshow, fue muy buena en esta población, con una $\chi^{2}$ de 2.2, p significativa, y valores similares a los de calificaciones específicas validadas para operaciones cardíacas ${ }^{30-33}$.

La capacidad de diferenciación de APACHE II en este estudio fue excelente, al obtener un valor de curva ROC de 0.92 (IC95\%, 0.88-0.96; $p=0.0001$ ). Si se compara con las curvas $\mathrm{ROC}$ registradas en otros protocolos que utilizaron el APACHE II en unidades de terapia intensiva generales, se advierte que es la de mayor área bajo la curva. En el trabajo original de Knaus, et al., la curva ROC fue de 0.86 , y fue similar a la observada en las cohortes de China (0.89), Canadá (0.86), Reino Unido (0.83) y Japón $(0.78)^{4,24-27}$. En comparación con los modelos específicos diseñados y validados para intervenciones cardíacas, la curva ROC de 0.92 que se obtuvo en esta investigación es aún la mejor (calificación ICNARC: 0.84; CASUS: 0.89 a 0.92; POCAS: 0.89; STS: 0.79; y EuroScore II: 0.78) ${ }^{13,30-35}$. 
En relación con el mejor valor de corte del sistema de calificación APACHE ॥ para predecir mortalidad intrahospitalaria, en el estudio actual se obtuvo a través de curva ROC un valor de 12. Este valor es importante, puesto que en la bibliografía existen pocos trabajos actuales que determinen el mejor valor de corte de la calificación para predecir episodios. Uno de ellos es el de Parajuli, et al., que comparó los sistemas APACHE II y IV en una terapia intensiva general. El punto de corte con el mejor valor del índice Youden fue de 17, algo mayor que el obtenido en dicho trabajo ${ }^{36}$. Kuhn, et al. evaluaron también la puntuación APACHE II según los mejores valores de corte en tres centros de cirugía cardíaca ${ }^{37}$. El valor de corte del APACHE II que indicó una mortalidad mayor de $50 \%$ fue de 28 , mayor al registrado en este protocolo.

Si se analizan los puntos finales secundarios, se reconoce que mayores valores de APACHE II se relacionaron también con mayor tasa de accidente cerebrovascular, sangrado quirúrgico, bajo gasto cardíaco y requerimiento de diálisis. En cualquier caso, no se conoce si la relación fue independiente y significativa, ya que el ajuste multivariado se llevó a cabo sólo para el episodio final primario. Al revisar las publicaciones especializadas, no se encontraron trabajos que predijeran estos sucesos a partir del APACHE II. Sin embargo, se han diseñado algunos estudios que utilizan dicho sistema en pacientes con diálisis. Entre ellos, Dobkin, et al. evaluaron la puntuación APACHE II para identificar los casos de mal pronóstico en una cohorte de pacientes que ingresaron a una unidad de terapia intensiva y que estaban bajo diálisis ${ }^{38}$. En la misma línea, Van Bommel, et al. estudiaron la aplicabilidad del sistema APACHE II en pacientes con insuficiencia renal aguda con necesidad de diálisis en una unidad de terapia intensiva quirúrgica ${ }^{39}$. Como resultado, el APACHE II calculado en las primeras 24 horas predijo mortalidad en esa población.

Como significancia clínica, la clasificación APACHE II es un modelo simple, validado en distintas cohortes internacionales, con más de 30 años de uso y con variables fisiológicas fáciles de calcular en la cama del paciente de cualquier unidad quirúrgica cardiovascular. Además, con los resultados de este estudio que demostraron una adecuada calibración y diferenciación en sujetos postoperatorios de procedimientos cardíacos, el APACHE II podría contribuir a estratificar el riesgo de esta población especial, y es por tanto el modelo de riesgo de elección.

El estudio tiene ciertas limitaciones que es preciso señalar. En primer lugar, es un diseño retrospectivo, por lo que eleva el riesgo de sesgos. En segundo lugar, el tamaño de la muestra es pequeño en relación con los modelos de riesgo evaluados en el plano internacional. Otra limitación a considerar es que se trató de un trabajo unicéntrico. De cualquier manera, se llevó a cabo en un centro monovalente cardiovascular de alta complejidad, con alto volumen de operaciones cardíacas.

\section{Conclusiones}

En este estudio, el sistema de calificación APACHE II demostró ser un predictor independiente de mortalidad intrahospitalaria en pacientes que cursan el postoperatorio de intervenciones quirúrgicas cardíacas en la unidad de terapia intensiva de un centro cardiovascular de alta complejidad, luego de mostrar una muy buena calibración y una excelente capacidad de diferenciación. El sistema APACHE II se relacionó además con mayor incidencia de bajo gasto cardíaco, ACV, sangrado quirúrgico y necesidad de diálisis, por lo que su utilización es atractiva para anticipar episodios en esta población especial de pacientes.

\section{Financiamiento}

Esta investigación no ha recibido ninguna beca específica de agencias de los sectores público, comercial, o sin ánimo de lucro.

\section{Conflicto de intereses}

Ninguno.

\section{Responsabilidades éticas}

Protección de personas y animales: Los autores declaran que para esta investigación no se han realizado experimentos en seres humanos ni animales.

Confidencialidad de los datos: Los autores declaran que han seguido los protocolos de su centro de trabajo sobre la publicación de datos de pacientes.

Derecho a la privacidad y consentimiento informado: Los autores declaran que en este artículo no se exponen datos de pacientes.

\section{Bibliografía}

1. Jones $\mathrm{HJ}$, de Cossart L. Risk scoring in surgical patients. $\mathrm{Br} \mathrm{J}$ Surg. 1999;86:149-57.

2. Rapsang AG, Shyam DC. Scoring systems in the intensive care unit: a compendium. Indian Journal of Critical Care Medicine: Peer-reviewed (Official Publication of Indian Society of Critical Care Medicine, India). 2014;18:220-8.

3. Sirio CA, Knaus WA. Application of prognostic scoring in adult intensive care. Curr Opin Anaesthesiol. 1990;3:241-4. 
4. Knaus WA, Draper EA, Wagner DP, Zimmerman JE. APACHE II: a severity of disease classification system. Crit Care Med. 1985 13(10):818-29.

5. Le Gall JR, Lemeshow S, Saulnier F. A new simplified acute physiology score (SAPS II) based on a European/North American multicenter study. JAMA. 1993;270(24):2957-63.

6. Moreno RP, Metnitz PG, Almeida E, Jordan B, Bauer P, Campor RA et al. SAPS 3: from evaluation of the patient to evaluation of the intensive care unit. Part 2: development of a prognostic model for hospital mortality at ICU admission. Intensive Care Med. 2005;31:1345-55.

7. Lemeshow S, Teres D, Klar J, Avrunin JS, Gehlbach SH, Papoport J. Mortality probability models (MPM II) based on an international cohort of intensive care patients. JAMA 1993;270:2478-86.

8. Lemeshow S, Le Gall JR. Modeling the severity of illness of ICU patients. A systems update. JAMA. 1994;272:1049-55

9. Ferreira FL, Bota DP, Bross A Mélot C, Vincent JL. Serial evaluation of the SOFA score to predict outcome in critically ill patients. JAMA 2001;286(14):1754-8.

10. Ceriani R, Mazzoni M, Bortone F, Gandini S, Solinas C, Susini G, et al Application of the sequential organ failure assessment score to cardiac surgical patients. Chest. 2003;123(4):1229-39.

11. Marshall JC, Cook DJ, Christou NV, Bernard GR, Sprung CL, Sibbald WJ. Multiple organ dysfunction score: a reliable descriptor of a complex clinical outcome. Crit Care Med. 1995;23(10):1638-52.

12. Turner JS, Morgan CJ, Thakrar B, Pepper JR. Difficulties in predicting outcome in cardiac surgery patients. Crit Care Med. 1995; 23(11):1843-50.

13. Nashef SA, Roques F, Sharples LD, Nilsson J, Smith C, Goldstone AR et al. EuroSCORE II. Eur J Cardiothorac Surg. 2012;41:734-44

14. Anderson RP. First publications from the Society of Thoracic Surgeons National Database. Ann Thorac Surg. 1994;57:6-7.

15. Ranucci M, De Toffol B, Isgró G, Romitti F, Conti D, Vicentini M. Hyperlactatemia during cardiopulmonary bypass: determinants and impact on postoperative outcome. Crit Care. 2006;10(6):167.

16. Demers $\mathrm{P}$, Elkouri S, Martineau R, Couturier A, Cartier R Outcome with high blood lactate levels during cardiopulmonary bypass in adult cardiac surgery. Ann Thorac Surg. 2000;70:2082-6.

17. Badreldin A, Doerr F, Elsobky S, Brehm BR, ABul-dahab M, Lehmann T, et al. Mortality prediction after cardiac surgery: blood lactate is indispensible. Thorac Cardiovasc Surg. 2013;61(8):708-17

18. Magilligan DJ, Teasdall R, Eisinminger R, Peterson E. Mixed venous oxygen saturation as a predictor of cardiac output in the postoperative cardiac surgical patient. Ann Thorac Surg. 1987;44 (3):260-2

19. Holm J, Håkanson E, Vánky F, Svedjeholm R. Mixed venous oxygen saturation predicts short- and long-term outcome after coronary artery bypass grafting surgery: a retrospective cohort analysis. $\mathrm{Br} \mathrm{J}$ Anaesth 2011;107(3):344-50

20. Ranucci M, Ballota A, Castelvecchio S, Baryshnikova E, Brozzi S, Boncilli $A$, et al. Intensive care unit admission parameters improve the accuracy of operative mortality predictive models in cardiac surgery. PLoS One. 2010;5(10):e13551

21. Knaus WA, Zimmerman JE, Wagner DP, Draper EA, Lawrence DE. APACHE - acute physiology and chronic health evaluation: a physiologically based classification system. Crit Care Med. 1981;9(8):591-7.

22. Jacobs S, Chang RWS, Lee B. One year's experience with the APACHE II severity of disease classification system in a general intensive care unit. Anaesthesia. 1987;42:738-44.
23. Jacobs S, Chang RWS, Lee B, Lee B. Audit of intensive care: a 30 month experience using the APACHE II severity of disease classification system. Int Care Med. 1988;14:566-74.

24. Sirio CA, Tajimi K, Knaus WA, Wagner DP, Hirasawa $H$, Sakanishi N, K et al. An initial comparison of intensive care in Japan and the United States. Crit Care Med. 1992;20:1207-15.

25. Rowan KM, Kerr JH, Major E, McPherson K, Shorta A, Vessey MP. Intensive care society's APACHE II study in Britain and Ireland. II: outcome comparisons of intensive careunits after adjustment for case mix by the American APACHE II method. BMJ. 1993;307(6910):977-81.

26. Teik E, Hutchinson MB, Short S, Buckley T, Lin E, Leung D. Verification of the acute physiology and chronic health evaluation scoring system in Hong Kong intensive care unit. Crit Care Med. 1993;21(5):698-705.

27. Wong DT, Crofts SL, Gómez M, McGuire GP, Byrick RJ. Evaluation of predictive ability of APACHE II system and hospital outcome in Canadian intensive care unit patients. Crit Care Med. 1995;23(7):1177-83.

28. Chassin MR, Galvin RW. The urgent need to improve health care quality. Institute of Medicine National Roundtable on Health Care Quality. JAMA. 1998;280:1000-5.

29. Blumenthal D, Epstein AM. Quality of health care. Part 6: the role of physicians in the future of quality management. $\mathrm{N}$ Engl $\mathrm{J}$ Med. 1996;335:1328-31

30. Hekmat K, Kroener A, Stuetzer H, Schwinger RH, Kampe S, Bennink GB, et al. Daily assessment of organ dysfunction and survival in intensive care unit cardiac surgical patients. Ann Thorac Surg. 2005;79(5):1555-62.

31. Hekmat K, Doerr F, Kroener A, Heldwein M, Bossert T, Badreldin AM, et al. Prediction of mortality in intensive care unit cardiac surgical patients. Eur J Cardiothorac Surg. 2010;38(1):104-9.

32. Exarchopoulos T, Charitidou E, Dedeilias P, Charitos C, Routsi C. Scoring systems for outcome prediction in a cardiac surgical intensive care unit: a comparative study. Am J Crit Care. 2015;24(4):327-34.

33. Tamayo E, Fierro I, Bustamante-Munguira J, Heredia-Rodríguez M, Jorge-Monjas $\mathrm{P}$, Maroto L, et al. Development of the post cardiac surgery (POCAS) prognostic score. Crit Care. 2013;17(5):R209.

34. Ariyaratnam P, Loubani M, Biddulph J, Moore J, Richards N, Chaudhry M, et al. Validation of the Intensive Care National Audit and Research Centre Scoring System in a UK adult cardiac surgery population. J Cardiothorac Vasc Anesth. 2015;29(3):565-9.

35. O'Brien SM, Shahian DM, Filardo G, Ferraris VA, Haan CK, Rich JB, et al. The Society of Thoracic Surgeons 2008 cardiac surgery risk models: part 2--isolated valve surgery. Ann Thorac Surg. 2009;88(1):23-42.

36. Parajuli BD, Shreshta GS, Pradhan B, Amatya R. Comparison of acute physiology and chronic health evaluation II and acute physiology and chronic health evaluation IV to predict intensive care unit mortality. Indian J Crit Care Med. 2015;19(2):87-91.

37. Kuhn C, Muller-Werdan U, Schmitt DV, Lange H, Pilz G, Kreuzer E, et al. Improved outcome of APACHE II score-defined escalating systemic inflammatory response syndrome in patients post cardiac surgery in 1996 compared to 1988-1990: the ESSICS-study pilot project. Eur J Cardiothorac Surg. 2000;17(1):30-7.

38. Dobkin J, Cutler R. Use of APACHE II classification to evaluate outcome of patients receiving hemodialysis in an intensive care unit. West $\mathrm{J}$ Med. 1988:149(5):547-50.

39. Van Bommel EF, Bouvy ND, Hop WC, Bruining HA, Weimar W. Use of APACHE II classification to evaluate outcome and response to therapy in acute renal failure patients in a surgical intensive care unit. Renal Failure. 1995;17(6):731-42. 